\title{
Light Coupling Between Photonic Crystal and Standard Photonic Waveguides in a Compact Photonic Integrated Circuitry Using 2D FDTD
}

\author{
S. Haxha ${ }^{*}, 1$, I. Dayoub ${ }^{3}$, F. AbdelMalek ${ }^{1}$, W. Aroua ${ }^{2}$, J. Trombi ${ }^{1}$ and H. Bouchriha ${ }^{2}$ \\ ${ }^{1}$ Photonics Group, Department of Electronics, University of Kent, Canterbury CT2 7NT, UK \\ ${ }^{2}$ National Institute of Applied Sciences and Technology, BP.676, Cedex 1080, Tunis, Tunisia \\ ${ }^{3}$ IEMN DOAE (UMR CNRS 8520) UVHC, Le Mont-Houy 59313 Valenciennes Cedex 9, France
}

\begin{abstract}
Finite difference time domain (FDTD) analysis was used to design a photonic integrated circuitry (PIC) based on a Silicon-on-Insulator (SOI) structure. Light coupling efficiency from a single mode photonic crystal waveguide (PCW) into a single mode fibre (SMF) through adiabatic tapered waveguide and microrings based on resonance acting as spot-size converters (SSCs), is demonstrated. Optimisation of each component and their integration into a single optical chip is presented. SSCs are optimised in order to reduce back reflection and increase coupling efficiency between all devices constituting the optical chip. Significant reduction of back-reflections, insertion losses and the Fabry-Perrot noise are obtained by optimising each component and when mode and impedance matching conditions are achieved. The transmission characteristics of each component are investigated and low propagation losses in the PIC are reported.
\end{abstract}

\section{INTRODUCTION}

Silicon-on-insulator (SOI) is a crucial material for future photonic integrated circuitry (PIC). Structures based on the SOI possess unique light properties owing to the large refractive index difference between silicon core layer (Si) (refractive index $\mathrm{n}=3.45$ ) and the $\mathrm{SiO}_{2}$ buried layer (refractive index $n=1.46$ ). These excellent light properties and compatibility with silicon Complimentary Metal Oxide Semiconductor (CMOS) $[1,2]$ integrated circuitry technology are highly promising for future PICs and for low-cost manufacturing. Due to maturity of CMOS manufacturing technologies, a very high refractive index contrast can be obtained in both vertical and horizontal directions by etching through the $\mathrm{Si}$ top layer. This permits the formation of submicron devices [3-6] which opens the way to low-cost PICs that can be mass-manufactured with CMOS technology on the same chip. In the last decades, various devices based on SOI such as cascaded Mach-Zehnder interferometers [7, 8], Arrayed Waveguide Gratings (AWGs) [9], directional couplers [10], photonic wire waveguides with core sizes of only $0.1 \mu \mathrm{m}^{2}$ [5] and bend radii as small as $1.0 \mu \mathrm{m}$ [6], have already been demonstrated. Additionally, various types of photonic crystal (PC) devices on SOI for future PIC applications, have also been demonstrated. PCs patterned into conventional semiconductors have achieved low losses for multimode straight PC waveguides (PCWs) [11]. It is well established that two dimensional (2D) slab PCs based on SOI [12], provide a high density integration of optical components, improve functionality and are cost efficient.

The PC technology can permit strong light confinement in compact structures [13-18] and can allow for innovative

*Address correspondence to this author at the Photonics Group, Department of Electronics, University of Kent, Canterbury CT2 7NT, UK; Tel: +44 (0) 1227 827257; Fax: +44 (0) 1227 456084; E-mail: S.Haxha@kent.ac.uk methods for manipulating the guided light. The prospect of PICs with integration density previously only considered possible with electronic circuits can be envisioned. High performance right angle bends and waveguide crossings that rely on resonances have been demonstrated in the photonic band gap (PBG) structures [19-21]. Integration of conventional waveguides and PCWs remains a challenging issue because of the mismatch mode widths. SOI devices, due to the high index contrast between the $\mathrm{Si}$ core and $\mathrm{SiO}_{2}$ claddings have a small mode size. Coupling of the PCWs into the SOI structures is associated with large losses due to the presence of the scattering losses in the PCWs. These limitations are the main challenges which prevent realisation of chip-to-chip integration of various PCWs and conventional waveguides on the SOI platform. Furthermore, coupling of the light from the single-mode fibres (SMF) into the optical chip (which consists of high density photonic integrated devices) will cause most of the light to be lost due to mode mismatching, and the difference in the refractive index between these devices will cause poor impedance matching [22]. Thus, large coupling losses are a direct result of small geometric overlaps and poor impedance matching of the optical modes between the PCW and SMF. Multiple mismatched interfaces between various components constituting the PIC would result in strong Fabry-Perot oscillations [23] in the transmission spectra. These oscillations can reach over $50 \%$ of the signal amplitude which can severely complicate the PIC. Therefore, it is essential to investigate advanced techniques and structures to integrate various devices in a compact PIC and overcome the above limitations.

In this study, we investigate a compact subsystem consisting of an integration of single mode PCW, an SOI rib waveguide, adiabatic tapered waveguide and microrings based on resonance acting as spot-size converters (SSCs) and a SMF. In this subsystem, the light beam propagating through the PCW enters into the SOI rib waveguide through 
the adiabatic tapered waveguide and then into SMF through microring resonators. Moreover, we optimise each device and investigate transmission and back reflection at each interfaces.

\section{THEORY}

In this study, the 2D finite difference time domain (FDTD) $[15-19,24]$ has been employed based on the Yee's algorithm [25]. In this numerical technique, the electric $\boldsymbol{E}(x$, $y)$ and magnetic $\boldsymbol{H}(x, y)$ field components are calculated at different grids having the same pitch but shifted over a half grid spacing, both in time and space. In this study, we have considered the 2D plane where the $z$ direction is normal to the $x-y$ plane of the grid. Time-dependent Maxwell's equations can be expressed as:

$$
\begin{aligned}
& \frac{\partial H}{\partial t}=-\frac{1}{\mu} \nabla \times E \\
& \frac{\partial E}{\partial t}=\frac{1}{\varepsilon} \nabla \times H-\frac{\sigma}{\varepsilon} E
\end{aligned}
$$

where $\boldsymbol{E}(\mathrm{V} / \mathrm{m})$ is the electric field, $\boldsymbol{H}(\mathrm{A} / \mathrm{m})$ is the magnetic field, $\varepsilon(\mathrm{F} / \mathrm{m})$ is the electrical permittivity, $\sigma(\mathrm{S} / \mathrm{m})$ is the electrical conductivity and $\mu(\mathrm{H} / \mathrm{m})$ is the magnetic permeability.

The magnetic and electric fields are derived from equations (1) and (2) at $\mathrm{t}=\mathrm{n} \Delta \mathrm{t}$ and $\mathrm{t}=(\mathrm{n}+1 / 2) \Delta \mathrm{t}$, respectively. Where $\mathrm{n}$ is integer and $\Delta \mathrm{t}$ is the time step related to the spatial step through the stability condition. A study of the FDTD method indicates that intensive memory and CPU time requirements mainly depend on two facts: first, the spatial increment step should be small in comparison with the smallest wavelength in order to make the numerical dispersion error negligible; secondly, $\Delta \mathrm{t}$ must be sufficiently small in order to satisfy the courant stability condition in $2 \mathrm{D}$. If the time step is not within this bound, the FDTD method will become numerically unstable, leading to an unbounded numerical error. In other words, the finite-difference algorithm requires that $\Delta \mathrm{t}$ to have a specific bound relative to the grid sizes $\Delta \mathrm{x}$ in $x$-direction and $\Delta \mathrm{y}$ in $y$-direction. This bound is necessary to avoid numerical instabilities. The computation region of the FDTD method must be limited in size. Maxwell's equations used inside the numerical domain must be modified on the outer boundary. In the FDTD method we employed as absorbing boundary condition the perfect matched layer (PML) proposed by Berenger [26]. The FDTD method represents one of the most powerful and computer efficient techniques that deals with electromagnetics. At the interface between structures (over one Yee cell in the numerical schema), we have employed the effective index method to calculate the components of the electric field and through equations (1) and (2) we have derived the magnetic field.

\section{RESULTS}

Our first step was to design a single mode PCW. The PC structure consists of a triangular lattice of air holes with radius of $0.48 a$, where $a$ is the lattice constant. The refractive index of the background is $\mathrm{n}=3.4$. The PC structure is composed of 13 rows and 9 columns. The PCW is constructed by removing one row of air holes in the $\Gamma-\mathrm{K}$ direction [27]. The waveguide width of the PCW is about $1.7 a$. We employed the plane-wave method [28] to calculate the mode dispersion. The dispersion diagram of the guided mode is illustrated in Fig. (1). It can be seen that the waveguide mode dispersion extends from $0.25 a / \lambda$ to $0.45 a / \lambda$.

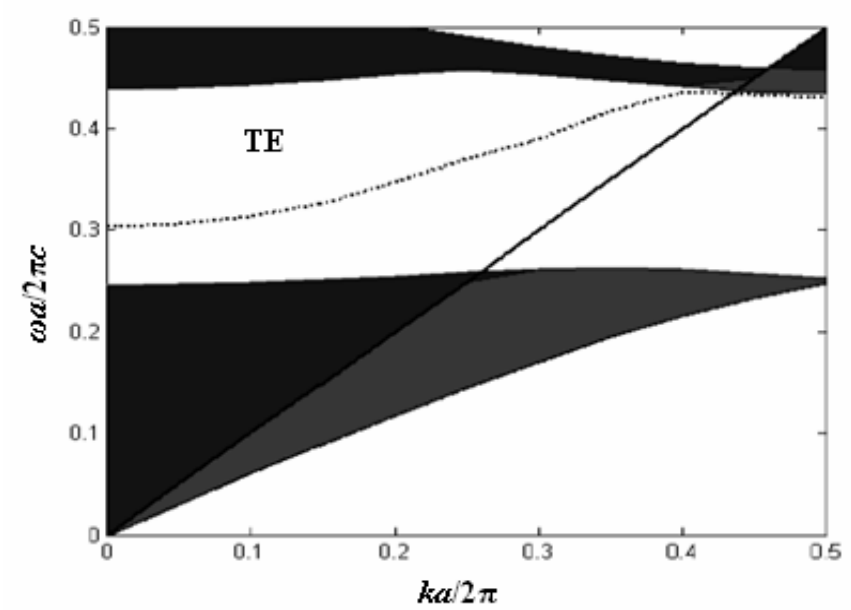

Fig. (1). Band diagram of guided mode.

Next, we investigated the integration of the PCW with a SOI rib waveguide. The SOI rib waveguide dimensions are as follows: core width, $\mathrm{W}=1.05$, core height, $\mathrm{H}=1.5 \mu \mathrm{m}$ and the Si layer height, $\mathrm{h}=0.6 \mu \mathrm{m}$ [29]. The SOI structure is deposited in a thick $\mathrm{SiO}_{2}$ layer. In order to effectively couple the light from the PCW into the SOI rib waveguide, we used a adiabatic tapered waveguide acting as a mode converter or a SSC. Schematic of the PCW, SSC and the SOI rib waveguide is illustrated in Fig. (2). Coupling efficiency between these components depends heavily on mode matching dimensions and matching conditions of the refractive index.

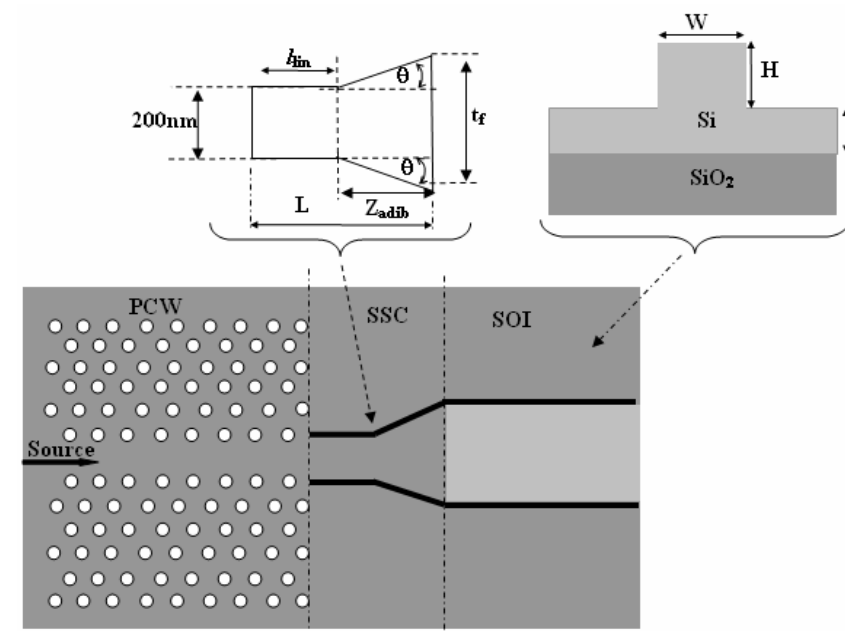

Fig. (2). Schematic of integration of PCW and SOI waveguide when SSC is incorporated.

Our simulations show that the effective refractive index of the SSC increases with the length; hence the SSC's length, can be adjusted to achieve matching conditions of the refractive index. However, there is a balance of how long the SSC can be. The length of the SSC should be optimised in order to keep low losses and to realise a compact PIC. The proposed SSC consists of two sections. First section (linear) has an initial width of $200 \mathrm{~nm}$ and a varying length $l_{\text {lin }}$. The second section consists of the adiabatic tapered waveguide structure with its varying length, $Z_{\text {adib }}$ and a final varying 
width $t_{f}$. The total length of the $\mathrm{SSC}$ is $\mathrm{L}=l_{\text {lin }}+\mathrm{Z}_{\text {adib. The }}$ following SSC parameters; $l_{\text {lin }}, Z_{\text {adiab }}$ and the tilt angle $\theta$, have all been optimised to achieve high coupling efficiency between the PCW and the SOI waveguide. A pulse of a 0.7 $\mu \mathrm{m}$ width with a Gaussian envelope in the time domain is launched in the PCW at $\mathrm{n}_{\mathrm{x}}=10$, and $\mathrm{n}_{\mathrm{y}}=20$ cells. The pulse is centred at $1.55 \mu \mathrm{m}$ operating wavelength, covering a wide range of frequencies. Here, we considered the transverse electric (TE) mode. The TE mode has the magnetic field parallel to the axes of the air holes. The electric field with $\boldsymbol{E}_{\mathrm{x}}$ and $\boldsymbol{E}_{\mathrm{y}}$ components in the plane and $\boldsymbol{E}_{\mathrm{z}}=0$ and $\boldsymbol{H}_{\mathrm{z}} \neq 0$ are considered.

Variations of the coupling efficiency and the reflected power as a function of the SSC adiabatic length $Z_{\text {adib }}$, when the tilted angle $\theta$ is equal to $1^{\circ}, 1.5^{\circ}$, and $2^{\circ}$, are illustrated in Fig. (3). Solid curves show the variation of the coupling efficiency. In this case, the SSC length $l_{\text {lin }}$ is kept fixed to $0.3 \mu \mathrm{m}$. The coupling efficiency was calculated by monitoring the electric field at a single cell in each device section. In other words, the ratio between the transmitted and incident fields is considered. It can be observed that the coupling efficiency increases as $Z_{\text {adib }}$ increases. Maximum coupling is achieved when $Z_{\text {adib }}=1.425 \mu \mathrm{m}$ and $\theta=1.5^{\circ}$. Our simulations clearly show that the coupling efficiency is very sensitive to the adiabatic length $Z_{\text {adib }}$ and the tilt angle $\theta$. Variation of the reflected power as a function of $Z_{\text {adib }}$ for the tilt angle equal to $1.0^{\circ}, 1.5^{\circ}$ and $2.0^{\circ}$, is illustrated by dashed curves on the right hand side of Fig. (3). As expected, the lowest reflected power is obtained when $\theta=1.5^{\circ}$. Thus, the optimised SSC dimensions; $l_{\text {lin }}=0.3 \mu \mathrm{m}, Z_{\text {adib }}=1.425 \mu \mathrm{m}$ and $\theta=1.5^{\circ}$, remained fixed throughout this study.

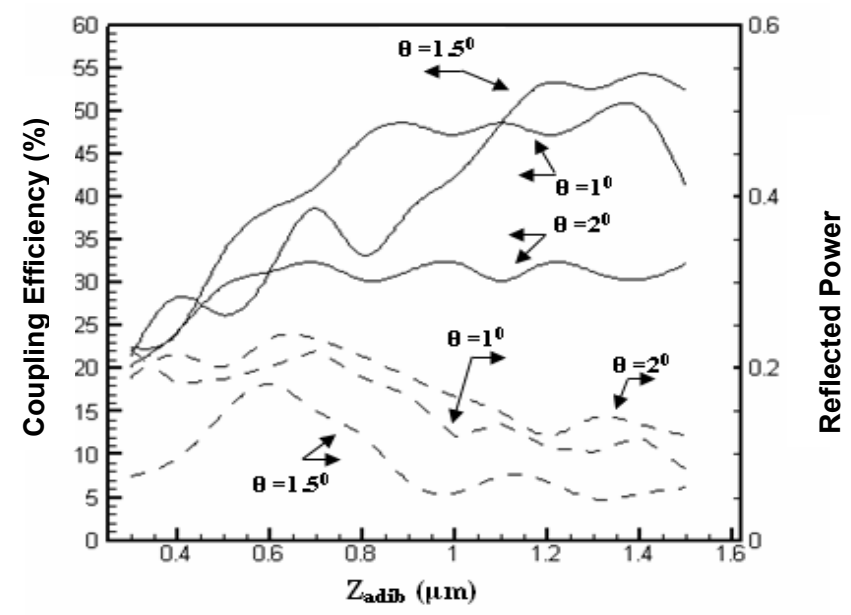

Fig. (3). Variation of the coupling efficiency and reflected power as a function of $Z_{\text {adib }}$ when $\theta=1^{0}, \theta=1.5^{\circ}$, and $\theta=2.0^{\circ}$.

Once the first SSC section is optimised, we focused on optimizing the second section. As mentioned above, the effective refractive index of the SSC varies with the length, thus, we investigated the effect of the SSC length $l_{\text {lin }}$ on the coupling efficiency and power reflection. Variations of the coupling efficiency and the reflected power as a function of the SSC adiabatic length $Z_{\text {adib }}$, when $l_{\text {lin }}$ is equal to $0.3 \mu \mathrm{m}$, $0.6 \mu \mathrm{m}$ and $1.2 \mu \mathrm{m}$, are presented in Fig. (4). Reflected power at the PCW/SSC interface is calculated by taking the Fourier transform of the pulses for each frequency [27]. The coupling efficiency is shown clearly by solid curves with its scale on the left-hand side, while the reflected power is shown by dashed curves with its scale on the right-hand side of this figure. One can see that the coupling efficiency increases as $l_{\text {lin }}$ decreases. The coupling efficiency increases significantly when $l_{\text {lin }}$ is $0.3 \mu \mathrm{m}$. Maximum coupling efficiency of $80 \%$ is achieved when $l_{\text {lin }}=0.3 \mu \mathrm{m}, \theta=1.5^{\circ}$ and $\mathrm{Z}_{\mathrm{adib}}=1.425 \mu \mathrm{m}$. One can see that the coupling efficiency slightly increases when $l_{\text {lin }}$ is equal to $0.6 \mu \mathrm{m}$ and $1.2 \mu \mathrm{m}$. In particular, for $l_{\text {lin }}=1.2 \mu \mathrm{m}$ and when $Z_{\text {adib }}$ is increased from $0.45 \mu \mathrm{m}$ to $1.5 \mu \mathrm{m}$, the coupling efficiency remained almost constant. Our simulations indicate that the SSC length $l_{\text {lin }}$, has higher impact on the coupling efficiency than the tilted angle $\theta$. Nevertheless, in order to achieve maximum coupling efficiency, it is necessary to optimise both parameters simultaneously, as shown in this study. The reflected power for all cases is calculated to be between 0.15 and 0.2 . As expected, the lowest reflected power is obtained when $l_{\text {lin }}=$ $0.3 \mu \mathrm{m}$.

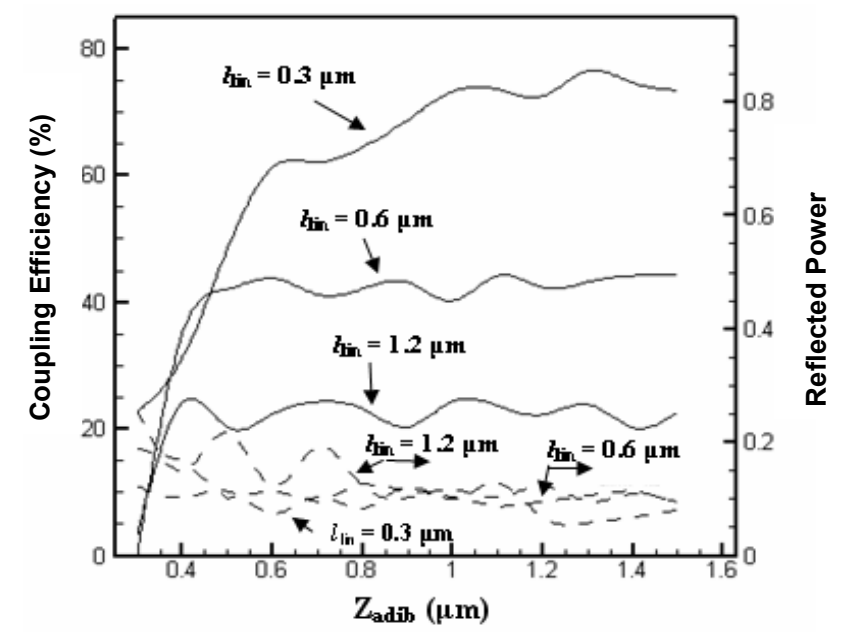

Fig. (4). Variation of the coupling efficiency and reflected power as a function of $Z_{\text {adib }}$ when $l_{\text {lin }}=0.3,0.6$, and $1.2 \mu \mathrm{m}$.

After the SSC dimensions were optimised, namely $l_{\text {lin }}=$ $0.3 \mu \mathrm{m}, Z_{\text {adiab }}=1.425 \mu \mathrm{m}$ and $\theta=1.5^{\circ}$, we focused on power reflections at the SSC/SOI interface for various SSC refractive indexes. Calculation of the power reflection as a function of the normalised frequency $a / \lambda$ when the refractive index of the SSC is 1.49 and 2.1, is illustrated in Fig. (5). Results for $n=1.49$ and $n=2.1$, are shown by solid and dashed curve, respectively. As can be observed from this figure, the power reflection is heavily dependent on the refractive index of the SSC and it varies with the normalised frequency $a / \lambda$. Low power reflections are obtained in the regions between $0.26 a / \lambda$ and $0.31 a / \lambda$. The lowest power reflection is achieved at peaks $0.28 \mathrm{a} / \lambda$ and $0.31 \mathrm{a} / \lambda$. When the normalised frequency increases from 0.31 to $0.4125 a / \lambda$, the power reflection increases rapidly. As can be observed from this figure, almost $50 \%$ of the reflected power is obtained when the normalized frequency exceeds $0.31 a / \lambda$. Therefore, in order to keep minimum power reflection and achieve maximum coupling efficiency at the same time, the following SSC fabrication parameters; $l_{\text {lin }}=0.3 \mu \mathrm{m}, Z_{\text {adiab }}=1.425 \mu \mathrm{m}, \theta$ $=1.5^{\circ}$ and $\mathrm{n}=2.1$, are keep fixed throughout this study at the normalized frequency equal to $0.28 \mathrm{a} / \lambda$. The snapshot of the electric field propagating from the PC through the SSC into the SOI waveguide is presented in Fig. (6). It is evident 
from this figure that there is a small amount of reflected power at the $\mathrm{SSC} / \mathrm{SOI}$ interface.

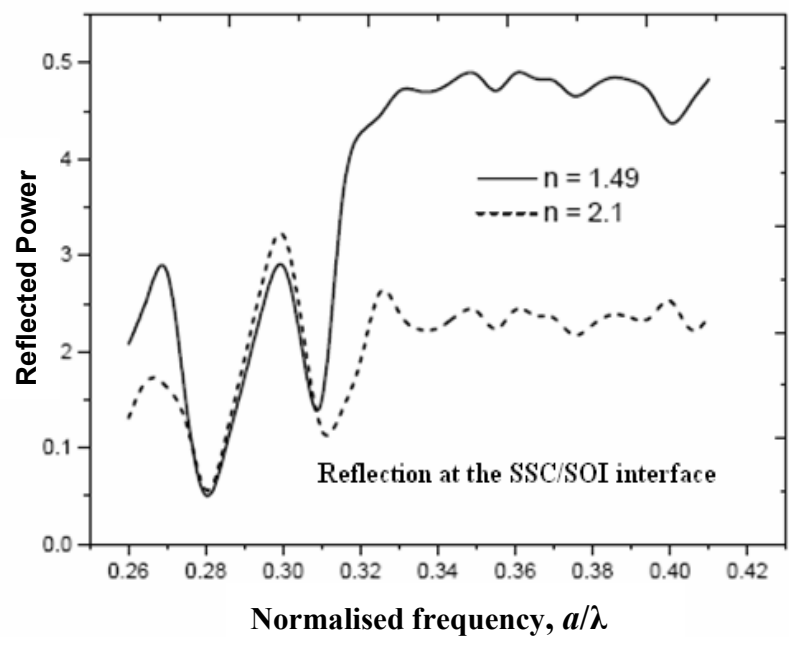

Fig. (5). Variation of the coupling efficiency as a function of $Z_{\text {adib }}$ when $\mathrm{n}=1.49$ and $\mathrm{n}=2.1$.

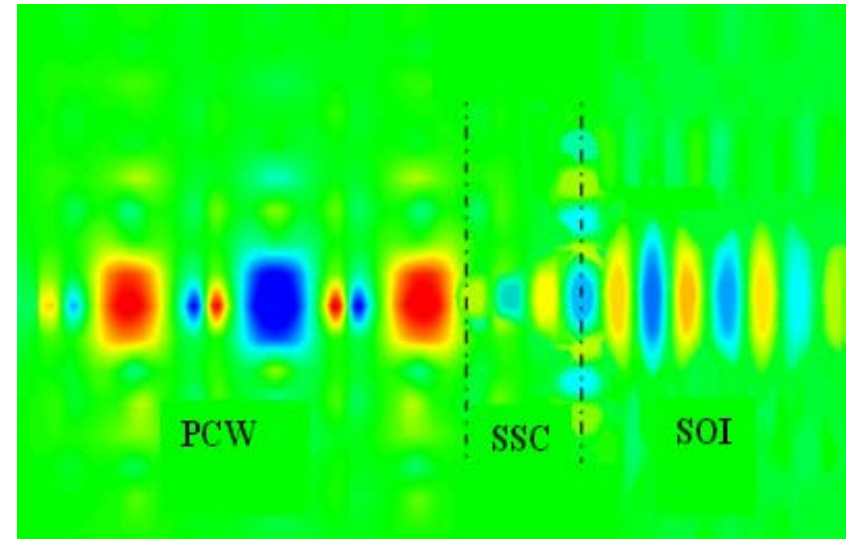

Fig. (6). Evolution of the electric field from the PC into the SOI waveguide at the normalised frequency equal to $0.28 \mathrm{a} / \lambda$, when $l_{\text {lin }}=$ $0.3 \mu \mathrm{m}, \mathrm{Z}_{\text {adib }}=1.425$ and $\theta=1.5^{\circ}$.

Once the light is effectively coupled from the PCW into the SOI waveguide, we investigate coupling of the light from the SOI waveguide into the SMF. The light source consists of a dipole, which creates pulses similar to the source in the $\mathrm{PCW}$, is placed at the entrance of the SOI waveguide.

The dipole creates pulses which propagate down the structure. To couple the light from the SOI into the SMF, a microring resonator is incorporated between the SOI waveguide and the SMF. Microring resonators are expected to play an essential role on achieving strong light confinement inside the ring waveguide and reduce the cavity size while maintaining high quality factor. Due to this, a single mode operation stability and spontaneous emission control, which can be reduced or cancelled, can be maintained [30]. A simple microring resonator consists of a ring waveguide as a resonant cavity closely coupled with one of two straight bus waveguides which can be used as an optical input and output device. Microring resonators are essential devices for realisation of PIC. The resonance is obtained when the round-trip phase acquired by the guided wave is equal to multiples of $2 \pi[31]$.
The extraction of light can be realised through evanescent manner, where by manipulating the evanescent field (for example forming a resonant system) extraction of light can be achieved. In this study, the microring resonator is designed as a couple of two rings with a common centre. The large ring has an optimum outer radius of $\mathrm{R}=4 \mu \mathrm{m}$, while the small (inner) ring has a varying anterior radius $r_{\text {inn }}$ filled with air $(\mathrm{n}=1)$. The construction of the microring structure is performed in $2 \mathrm{D}$ by considering an equivalent refractive index in the $z$-direction [32]. The refractive index $\mathrm{n}_{\mathrm{eq}}$ was calculated in the plane, independently of the structure, by taking $\mathrm{n}_{\mathrm{eq}}=\beta / k_{0}$, where $\beta$ is the propagation constant and $k_{0}$ is wave number in the vacuum. The microring resonator has a thickness $\mathrm{t}=280 \mathrm{~nm}$ and a refractive index $\mathrm{n}=1.45$, as shown in Fig. (7). In other words, a nanowire waveguide ring based on a resonance, is employed to couple the light from the SOI into the SMF.

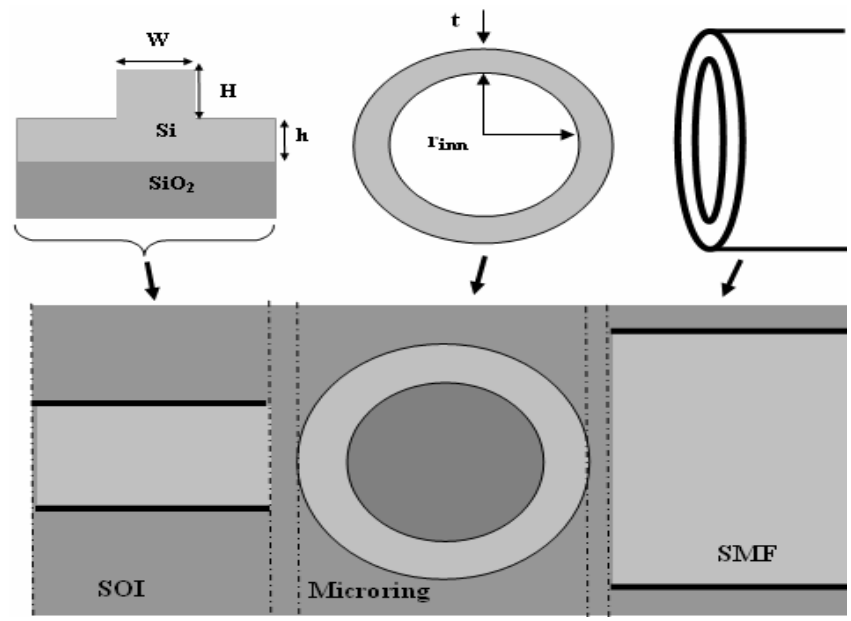

Fig. (7). Schematics of integration of the SOI waveguide, microring and SMF.

Due to the resonant tunnelling effects, we placed the Gaussian source in the SOI slab entrance and then coupled it into the microring. In this way, by forming a resonant system, we've amplified the light in the SOI waveguide and the microring. Due to the computer memory limitations, we simplified the SOI structure, where the wave vector in the $z$ direction is considered and in the third direction, the refractive index is assumed to be constant.

Since the light coupling into the microring resonator depends heavily on the gap separation between the microring, the SOI waveguide and the SMF, we focused on the investigation of inner and outer radius effects on the coupling efficiency. Calculation of the coupling efficiency as a function of the inner radius $r_{\text {inn }}$, when the outer radius $R$ is equal to 4 and $4.5 \mu \mathrm{m}$ is illustrated in Fig. (8). Results for $\mathrm{R}=4 \mu \mathrm{m}$ and $\mathrm{R}=4.5 \mu \mathrm{m}$ are shown by solid and dashed curve, respectively.

Maximum coupling efficiency of $82 \%$ is obtained when $\mathrm{R}=4 \mu \mathrm{m}$ and $\mathrm{r}_{\mathrm{inn}}=0.8 \mu \mathrm{m}$. Other values of $\mathrm{R}$ were also investigated, however, the coupling efficiency was significantly lower compared to when $\mathrm{R}=4 \mu \mathrm{m}$. At the $\mathrm{SOI} /$ microring interface, back reflection of the light is around 0.15 . Our simulations indicate that the maximum coupling efficiency between the SOI waveguide and the 


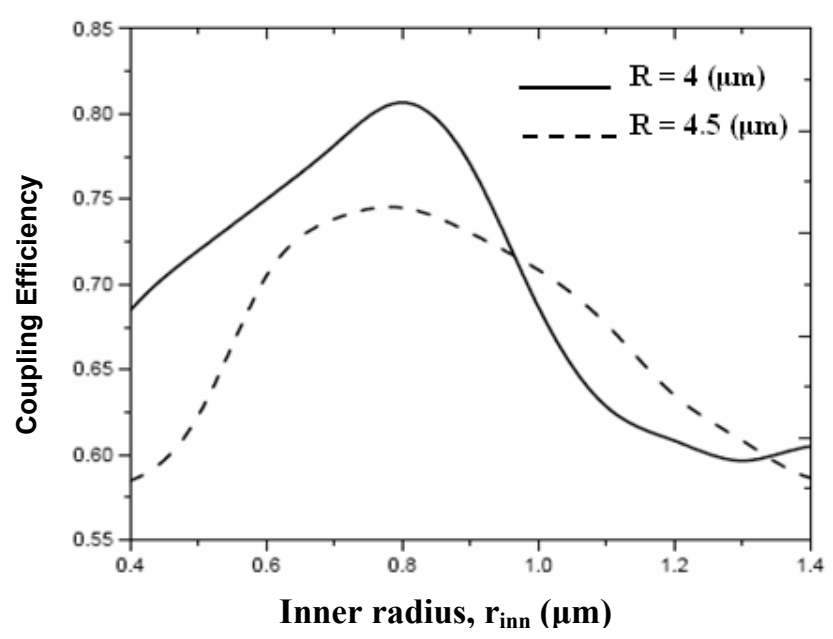

Fig. (8). Variation of the coupling efficiency as a function of the inner radius $r_{\text {in }}$ when $R=4 \mu \mathrm{m}$ and $4.5 \mu \mathrm{m}$.

microring can be significantly increased, when both inner and outer radii are optimised, while maintaining the resonance. The snapshot of the electric field propagating from the microring into the SMF, is presented in Fig. (9). In this case, the gap between the microring and the SMF is around $150 \mathrm{~nm}$.

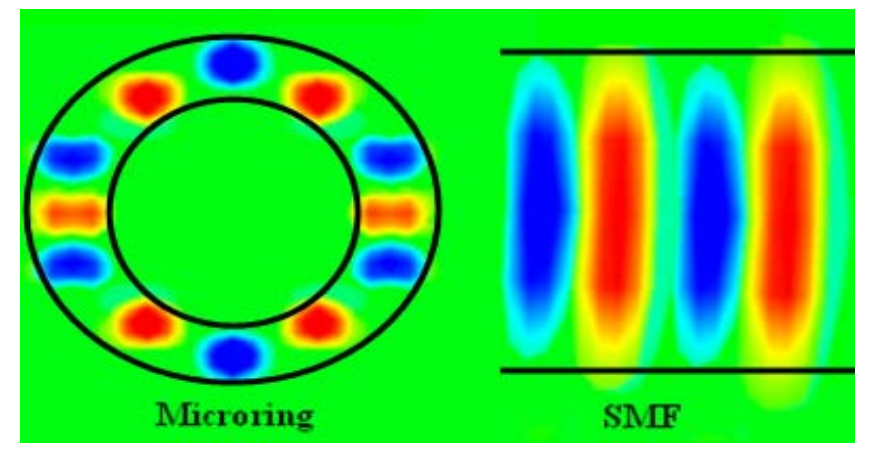

Fig. (9). Evolution of the electric field from the microring into the $\mathrm{SMF}$, when $\mathrm{R}=4 \mu \mathrm{m}$ and $\mathrm{r}_{\mathrm{inn}}=0.8 \mu \mathrm{m}$.

Transmission of the light, calculated at the SOI waveguide output and at the microring/SMF interface, as a function of the normalised frequency $a / \lambda$, is presented in Fig. (10). Solid and dashed curves represent the light transmission at the SOI waveguide output and microring/SMF interface, respectively. One can see, that the light transmission increases as the normalised frequency $a / \lambda$ increases. At the SOI waveguide output, a maximum transmission of about $80 \%$ is achieved at normalised frequency $0.372 a / \lambda$. However, due to insertion losses, transmission at the microring/SMF drops to $68 \%$. From Fig. (10), it can be observed that reasonably small oscillations are present in both cases. Due to the optimisation of each device, where mode and impedance matching is achieved, the amplitude of these oscillations are reduced significantly. Consequently, the FabryPerrot noise is small. The impedance matching contributes also to reduce scattering losses. It is relevant to note that the Fabry-Perrot noise [33], is related to the nature of oscillations in the either transmission or reflection spectra. Poor matching interfaces in the PIC introduce strong Fabry-Perrot oscillations in the transmission spectra, which can be around $50 \%$ of the signal amplitude [34].

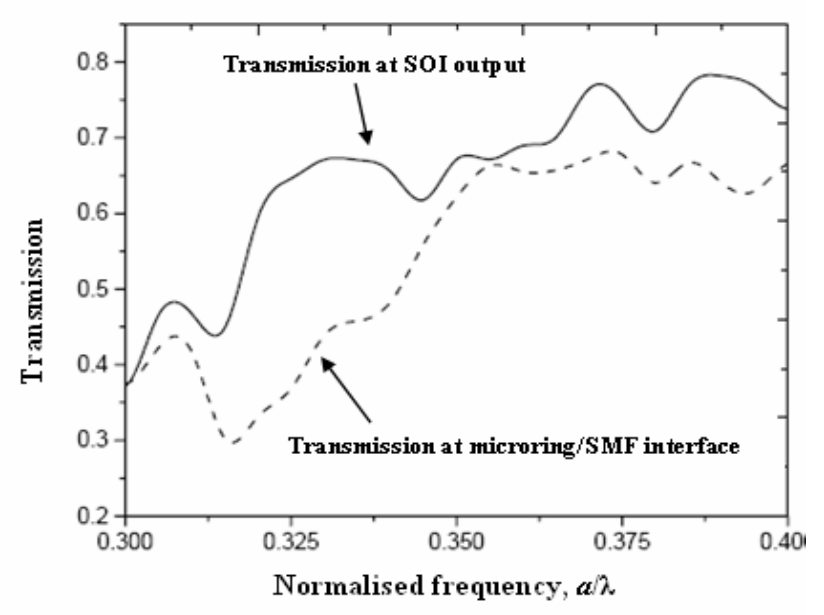

Fig. (10). Variation of the light transmission, at the SOI output and microring/SMF interface, as a function of the normalized frequency.

Transmission of the light, calculated at the SOI waveguide output and at the microring/SMF interface, as a function of the normalised frequency $a / \lambda$ when $n=1.49$ and $a / \lambda=0.37$ (impedance matching is not achieved), is illustrated in Figs. $(\mathbf{1 1}, \mathbf{1 2})$, respectively. From these figures, it can clearly be seen that oscillations are significantly higher compared to those presented in Fig. (10), when matching was achieved for $\mathrm{n}=2.1$.

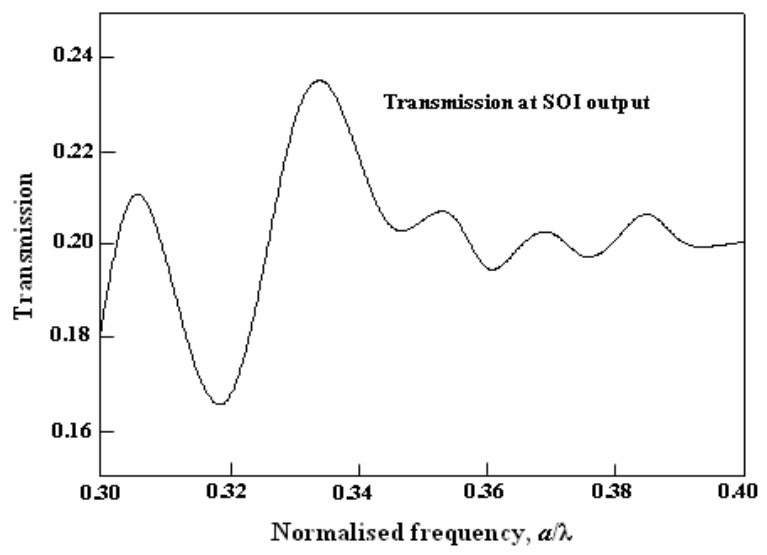

Fig. (11). Variation of the light transmission, at the SOI output as a function of the normalized frequency, when impedance matching is not achieved.

It is relevant to note that the oscillations in the spectra are high. These oscillations are due to the mismatching of the impedance. In other words the matching of the refractive index on both sides is not achieved; resulting in poor overlapping between the modes, as a consequence, oscillation amplitude in the transmission spectra is increased.

The motivation for this study was to design a low loss, compact and complex PIC. In many photonic device applications, complex PICs are unavoidable and transmission of light in such circuits is not trivial task. Therefore, in this study, we intentionally increased the complexity of the PIC by adding an extra microring. The added microring has similar dimensions to the first (optimised) microring. Schematic 
of the structure consisting of the SOI coupled to two microrings separated by an air gap g and butt-coupled to the SMF, is illustrated in Fig. (13). It is worth mentioning that when the second microring is added, a coupled resonant system is achieved. Our simulations indicate that the light coupling between the microrings show interesting behaviour when the air gap $g$ varies.

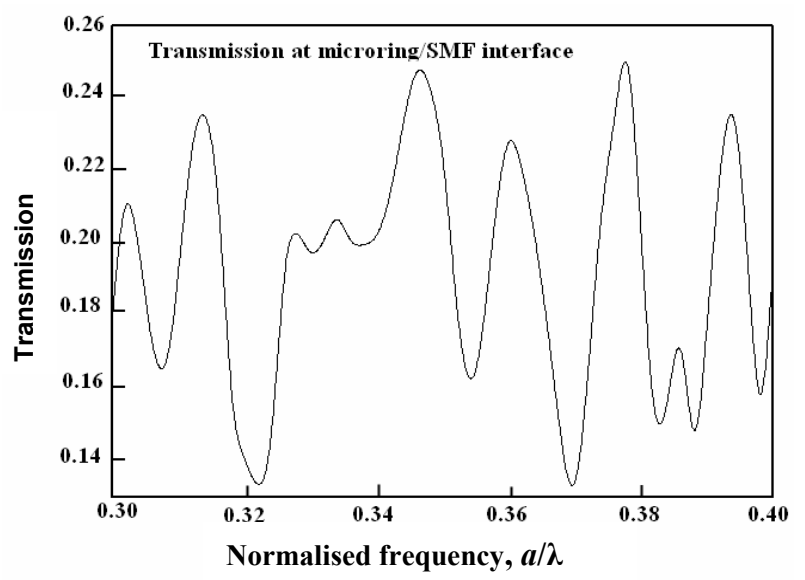

Fig. (12). Variation of the light transmission, at the microring/SMF interface, as a function of the normalized frequency, when impedance matching is not achieved.

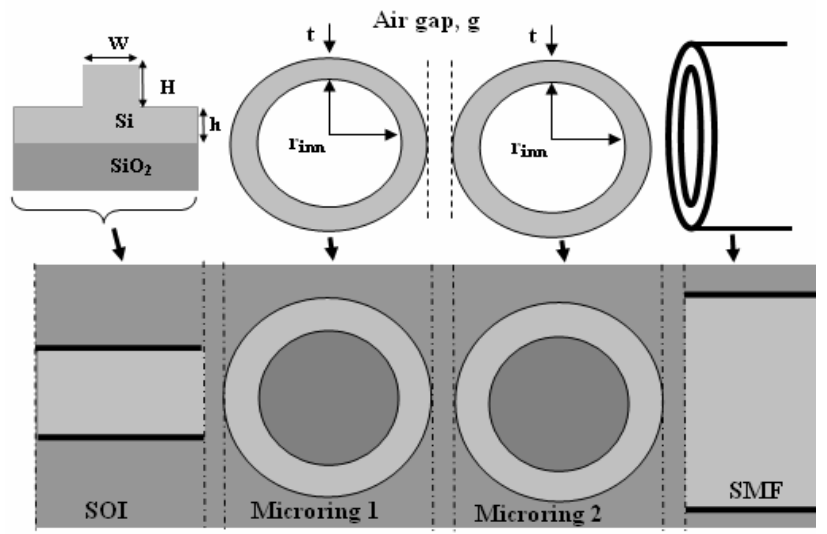

Fig. (13). Schematic of two microrings used as a resonant system coupled to the SMF.

Variation of the coupling efficiency as a function of the air gap, when the inner radius of both microrings is fixed to $0.8 \mu \mathrm{m}$, is illustrated in Fig. (14). Results of the coupling efficiency for both outer microring radii equal to $4 \mu \mathrm{m}$ and $4.5 \mu \mathrm{m}$, are illustrated by dashed and solid curves, respectively. We can see that as the gap between the microrings increases the coupling efficiency decreases. Maximum coupling efficiency is achieved when microrings are attached to each other for $\mathrm{R}=4.5 \mu \mathrm{m}$. However, one can see that when the outer radius of both microrings is reduced from $4.5 \mu \mathrm{m}$ to $4 \mu \mathrm{m}$ and the air gap is increased to $\mathrm{g}=120 \mathrm{~nm}, 95 \%$ of the coupling efficiency is realised. This indicates that we can still achieve a higher coupling efficiency than $95 \%$ and reduce the device radius by $500 \mathrm{~nm}$. The snapshot of the electric field propagating from the first microring through the second microring into the SMF when $\mathrm{g}=120 \mathrm{~nm}$, is presented in Fig. (15). It can clearly be noticed that the light is very well confined in the SMF, which shows that the microring based resonant system, has effectively coupled the light from the SOI into the SMF. Schematics of integration of PCW, SSC, SOI rib waveguide, microrings and SMF is illustrated in Fig. (16). The electric field pattern along the entire optical chip, is illustrated in Fig. (17). One can see that the light is well confined along the entire system, despite the optical interfaces. The microring fabrication parameters are optimised by choosing desired shapes in terms of reducing back reflections and minimising oscillations in the transmission spectra.

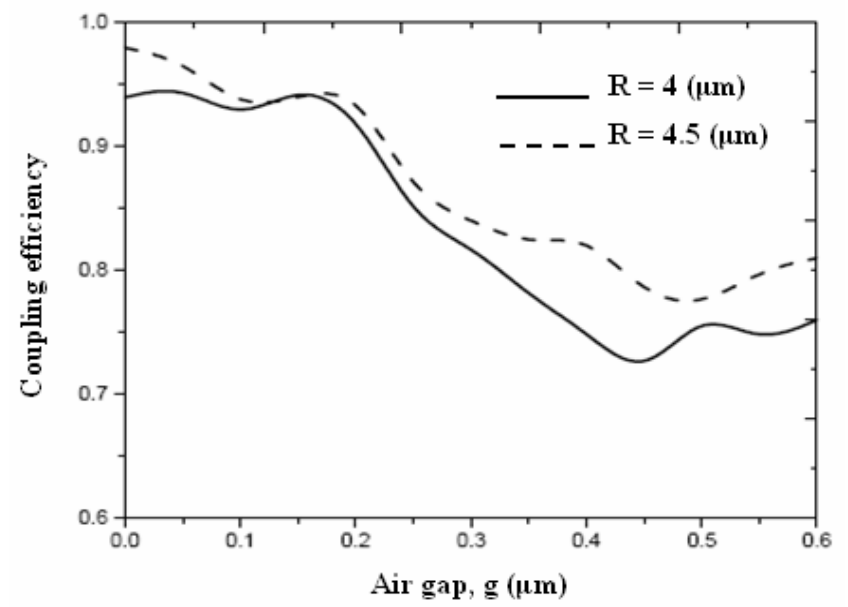

Fig. (14). Variation of the coupling efficiency as a function of the air gap between two microrings.

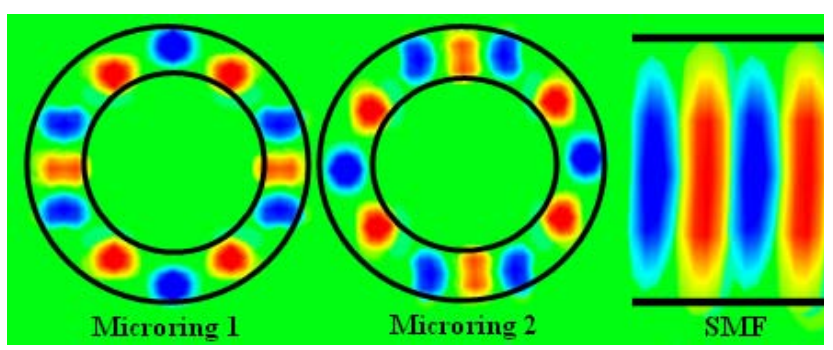

Fig. (15). Electric field evolution along two microrings and the SMF.

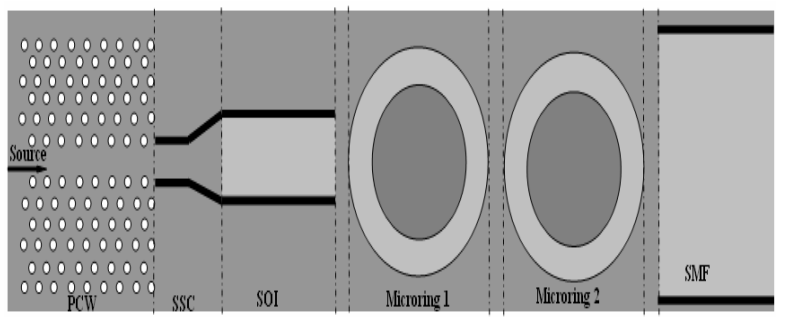

Fig. (16). Schematics of integration of PCW, SSC, SOI rib waveguide, microrings and SMF.

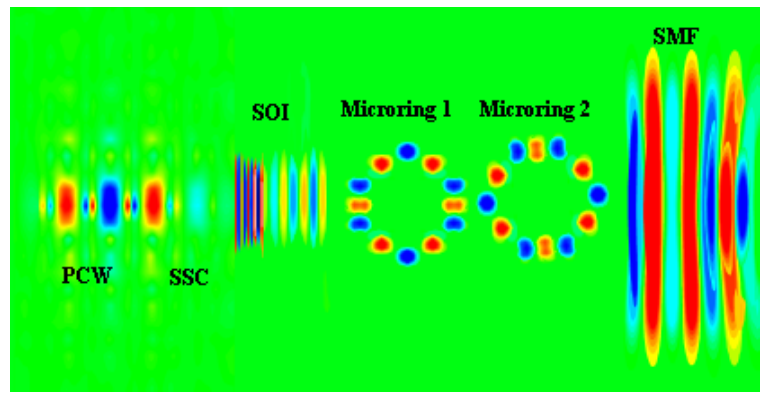

Fig. (17). Electric field pattern along the entire system. 
Variation of light transmission along the chip length, from the PCW into the SMF, through the taper, SOI waveguide and both microrings, is illustrated in Fig. (18). One can see that the light transmission decreases as it propagates along the optical chip.

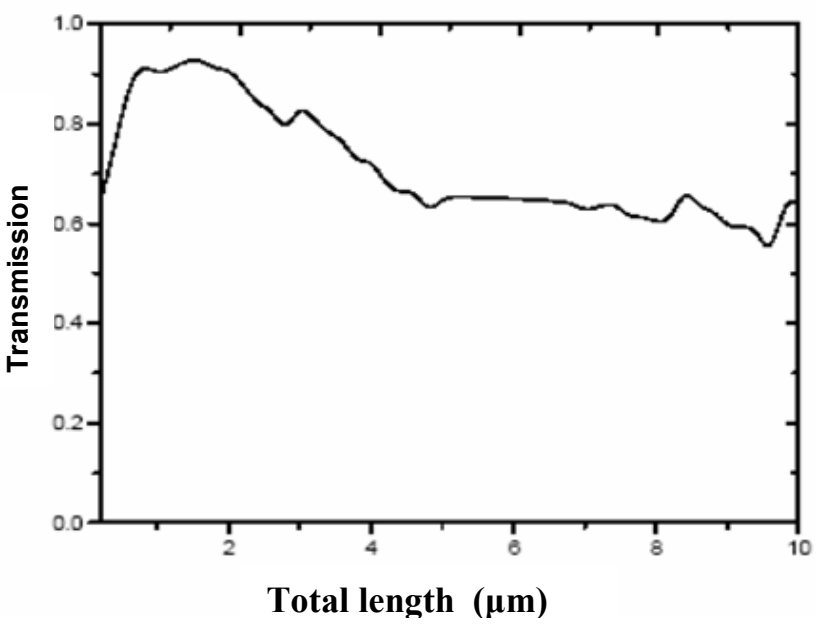

Fig. (18). Transmission at the entrance of the SMF.

The transmission of light remains constant when the propagating length exceeds $10 \mu \mathrm{m}$. The total length is about $20 \mu \mathrm{m}$ and for this length, the light transmission decreases at the minimum of $58 \%$. The Gaussian beam waist is broadened by the use of appropriate dimensions of microrings, which fit the core size into the SFM. It is evident that, the two microrings form a resonant system that can be used as a SSC to significantly enhance the coupling efficiency. It is relevant to note that, by carefully optimizing each device and using desired SSCs, the light can be efficiently coupled from the PCW into the SMF, regardless of the huge difference between the cross-sections of the optical modes. The overall output light transmission in the SMF is around $58 \%$. Considering the fact that various devices with different dimensions are integrated into a compact optical chip, this transmission of $58 \%$ could be considered significant.

\section{CONCLUSION}

In conclusion, by using two dimensional (2D) finite difference time domain (FDTD) computational technique, we have presented a photonic integrated circuitry (PIC) consisting of the integration of a single mode photonic crystal waveguide (PCW), SOI waveguide, a adiabatic tapered waveguide and microrings based on resonance, acting as spot-size converters (SSCs), and a SMF. A high coupling efficiency between devices constituting the PIC has been realized by incorporating optimised tapered waveguide and microring resonators. It is demonstrated that, in order to reduce back-reflections, insertion losses and the Fabry-Perrot noise, optimisation of each component, and optical mode matching and impedance matching condition is necessary. Furthermore, it is demonstrated that such design can be used to effectively integrate various photonic components in a complex optical chip within a compact area. It should be noted that additional losses such as scattering losses due to imperfect fabrication have not been taken into account. Further work will be investigating the PIC light coupling in 3D using 3D FDTD.

\section{REFERENCES}

[1] Bogaerts W, Baets R, Dumon P, et al. Nanophotonic waveguides in silicon-on-insulator fabricated with CMOS Technology. IEEE J Lightwave Technol 2005; 23: 401-12.

[2] Jalali B, Yegnanarayana S, Yoon T, Yoshimoto T, Rendine I, Coppinger F. Advanced in Silicon-on-insulator optoelectronics. IEEE J Selected Top Quantum Electron 1998; 4: 938-47.

[3] Sakai A, Hara G, Baba T. Propagation characteristics of ultra-high $\Delta$ optical waveguide on silicon-on-insulator substrate. Jpn J Appl Phys 2001; 40: L383-L385.

[4] Taillaert D, Bogaerts W, Bienstman P, Zutter DD, Baets R. $3^{\text {rd }}$ Workshop on Photonic and Electromagnetic Crystal Structures (PECS3), St. Andrews: Scotland 2001.

[5] Dumon P, Roelkens G, Bogaerts W, et al. Basic photonic wire components in silicon-on-insulator. In: Proc. $2^{\text {nd }}$ IEEE Int Conf Group IV Photon 2005; pp. 189-91.

[6] Bogaerts W, Dumon P, Van Thourhout D, et al. Compact wavelength-selective functions in silicon-on-insulator photonic wires. IEEE J Selected Top Quantum Electron 2006; 12: 1394-401.

[7] Trinh PD, Yegnanarayanan S, Coppinger F, Jalali B. Silicon-oninsulator (SOI) phased-array wavelength multi-demultiplexer with extremely low-power sensivity. IEEE Photon Technol Lett 1997; 9: 940-2.

[8] Sofer RA, Bennett BR. Electrooptic effects in silicon. IEEE J Quantum Electron 1987; 23: 123-9.

[9] Ng W, Walston AA, Tangoman GL, Lee JJ. Newberg IL, Bernstein $\mathrm{N}$. The first demonstration of an optically steered microwave phased array antenna using tru-time delay. IEEE J Lightwave Technol 1991; 9:1124-31.

[10] Trinh PD, Yegnanarayanan S, Jalali B. Integrated optical directional couplers in silicon-on-insulator. Electron Lett 1995; 13: 2097-8.

[11] Talneau A, Le Gouezigou L, Bouadma N. Quantitative measurement of low propagation losses at $1.55 \mu \mathrm{m}$ on planar photonic crystal waveguides. Opt Lett 2001; 26: 1259-61.

[12] Bogaerts W, Wiaux V, Taillaert D, et al. Fabrication of photonic crystals in silicon-on-insulator using 248-nm deep UV lithography. IEEE J Selected Top Quantum Electron 2002; 8: 928-34.

[13] Yablonovitch E. Inhibited spontaneous emission in solid-state physics and electronics. Phys Rev Lett 1987; 58: 2059-62.

[14] John S. Strong localization of photons in certain disordered dielectric superlattices. Phys Rev Lett 1987; 58: 2486-9,

[15] AbdelMalek F, Belhadj W, Haxha S, Bouchriha H. Realization of high coupling efficiency by employing a concave lens based on 2D photonic crystals with negative refractive index. IEEE J Light Technol 2007; 25: 3168-74.

[16] Haxha S, Belhadj W, AbdelMalek F, Bouchriha H. Analyses of wavelength demultiplexer based on photonic crystals. IEEE Proc Optoelec 2005; 152: 193-8.

[17] Belhadj W, Gamra D, AbdelMalek F, Haxha S, Bouchriha H. Design of 2D photonic crystal structure based in all-angle negative refractive effect for application in focusing systems. IET Proc Optoelec 2007; 1: 91-5.

[18] Aroua W, Ouerghi F, Haxha S, et al. Analysis and optimisation of high density photonic crystal devices in a subsystem by use of finite difference time domain. IET Proc Optoelec 2008; 2: 10-5.

[19] Mekis A, Chen JC, Kurland I, Fan I, Villeneuve PR, Joannopoulos JD. High transmission through sharp bends in photonic crystal waveguides. Phys Rev Lett 1996; 77: 3787-90.

[20] Nakamura H, Sugimoto Y, Kanamoto Y, et al. Ultra-fast photonic crystal/quantum dot all-optical switch for future photonic networks. Opt Express 2004; 12: 6606-14.

[21] Johnson SG, Manolatou C, Fan S, Villeneuve PR, Joannopoulos JD, Haus HA. Elimination of cross talk in waveguide intersections. Opt Lett 1998; 23: 1855-7.

[22] Sakoda K. Optical transmittance of a two-dimensional triangular photonic lattice. Phys Rev B 1995; 51: 4672-5.

[23] Karle TJ, Brown DH. Wilson R, Steer M, Krauss TF. Planar photonic crystal cavity waveguides. IEEE J Quantum Electron 2002; 8: 909-18.

[24] Taflove A. Computational Electrodynamics. The Finite Difference Time Domain Method. Artech House 1995.

[25] Yee SK. Numerical solution of initial boundary value problems involving Maxwell's equations in isotropic media. Trans Antenna Propag 1966; 14: 302-7. 
[26] Berenger JPA. Perfectly matched layer for the absorption of electromagnetic waves. J Comput Phys 1994; 114: 185-200.

[27] Mekis A, Chen JC, Kurland I, Fan S, Villeneuve PR, Joannopoulos JD. High transmission through sharp bends in photonic crystal waveguides. Phys Rev Lett 1996; 77: 3787-90.

[28] Leung KM, Liu YF. Full vector wave calculation of photonic band structures in face-centered-cubic dielectric media. Phys Rev Lett 1990; 65: 2646-9.

[29] Seong PC, Ching EP, Soon TL, Graham Reed T, Vittorio MNP. Single-mode and polarization-independent silicon-on-insulator waveguides with small cross section. IEEE J Lightwave Technol 2005; 23: 2103-11.
[30] Baba T. Photonic crystals and microdisk cavities based on GaInAsP-InP system. IEEE J Selected Top Quantum Electron 1997; 3: 808-30.

[31] Nishihara H, Haruna M, Suhara T. Optical Integrated Circuits. New York: McGraw-Hill 1989; pp. 43-4.

[32] Sakai A, Baba T. FDTD simulation of photonic devices and circuits based on circular and fan-shaped microdisks. IEEE J Lightwave Technol 1999; 17: 1493-9.

[33] Talneau A, Lalanne P, Agio M, Soukoulis CM. Low-reflection photonic-crystal taper for efficient coupling between guide sections of arbitrary widths. Opt Lett 2002; 27: 1522-4.

[34] Karle TJ, Brown DH, Wilson R, Steer M, Krauss TF. Planar photonic crystal coupled cavity waveguides. IEEE J Quantum Electron 2002; 8: 909-18.

(C) Haxha et al.; Licensee Bentham Open.

This is an open access article licensed under the terms of the Creative Commons Attribution Non-Commercial License (http://creativecommons.org/licenses/by$\mathrm{nc} / 3.0 /$ ) which permits unrestricted, non-commercial use, distribution and reproduction in any medium, provided the work is properly cited. 